\title{
THE APPROXIMATION OF GONTINUOUS FUNGTIONS BY RIESZ TYPICAL MEANS OF THEIR FOURIER SERIES
}

B. KWEE

(Received 26 July 1966)

\section{Introduction}

Let $f(x)$ be a continuous function with period $2 \pi$. It is well known that the Fourier series of $f(x)$ is summable Riesz of any positive order to $f(x)$. The aim of this paper is the proof of the following theorem.

THEOREM A. If $f(x)$ is a continuous function with period $2 \pi, k$ is a positive integer,

$$
f(x) \sim \frac{1}{2} a_{0}+\sum_{\nu=1}^{\infty}\left(a_{\nu} \cos \nu x+b_{\nu} \sin v x\right)=\sum_{\nu=0}^{\infty} s_{\nu}(x)
$$

and

$$
R_{\lambda}^{k}(x)=\sum_{\nu \leqq \lambda}\left(1-\frac{\nu}{\lambda}\right)^{k} s_{\nu}(x)
$$

then

$$
R_{\lambda}^{k}(x)-f(x)=\frac{k}{\pi} \int_{a}^{\infty} \frac{\phi_{x}(t / \lambda)}{t^{2}} d t+O\left(\omega_{2}\left(\frac{1}{\lambda}, f\right)\right)
$$

where

$$
\begin{aligned}
\phi_{x}(t) & =f(x+t)+f(x-t)-2 f(x), \\
\omega_{2}(h, f) & =\sup _{|\delta| \leqq h}|| \phi_{x}(\delta) \|=\sup _{|\delta| \leqq h} \max _{x}\left|\phi_{x}(\delta)\right| .
\end{aligned}
$$

\section{Lemmas}

In the proof of the following lemmas, we assume that $n=[\lambda]$.

LEMMA 1.

$$
\int_{1}^{\infty} \phi_{x}\left(\frac{t}{\lambda}\right) \frac{\cos t}{t^{2}} d t=O\left(\omega_{2}\left(\frac{1}{\lambda}, t\right)\right)
$$


Proof.

$$
\begin{aligned}
\int_{1}^{\infty} \phi_{x}\left(\frac{t}{\lambda}\right) & \frac{\cos t}{t^{2}} d t=\int_{1}^{n \pi} \phi_{x}\left(\frac{t}{\lambda}\right) \frac{\cos t}{t^{2}} d t+\sum_{v=1}^{\infty} \int_{\nu n \pi}^{(\nu+1) n \pi} \phi_{x}\left(\frac{t}{\lambda}\right) \frac{\cos t}{t^{2}} d t \\
& =\frac{1}{n} \int_{1 / n}^{\pi} \phi_{x}\left(\frac{n t}{\lambda}\right) \frac{\cos n t}{t^{2}} d t+\frac{1}{n} \int_{0}^{\pi} \cos n t \sum_{\nu=1}^{\infty} \frac{(-1)^{n \nu} \phi_{x}\left(\frac{v n \pi+n t}{\lambda}\right)}{(v \pi+t)^{2}} d t \\
& =A_{n}+B_{n} .
\end{aligned}
$$

Suppose that $P_{n}(x)$ is the best approximation trigonometric polynomial of order $n$ to $f(x)$. Then [2]

$$
E_{n}(f)=\left\|f(x)-P_{n}(x)\right\|=O\left(\omega_{2}\left(\frac{1}{n}, f\right)\right)
$$

and

$$
\left\|P_{n}^{\prime \prime}(x)\right\|=O\left(n^{2} \omega_{2}\left(\frac{1}{n}, f\right)\right)
$$

Hence

$$
\phi_{x}(t)=\psi_{x}(t)+O\left(\omega_{2}\left(\frac{1}{n}, f\right)\right)
$$

where

$$
\psi_{x}(t)=P_{n}(x+t)+P_{n}(x-t)-2 P_{n}(x)
$$

Now

$$
\begin{aligned}
A_{n}= & \frac{1}{n} \int_{1 / n}^{\pi} \phi_{x}\left(\frac{n t}{\lambda}\right) \frac{\cos n t}{t^{2}} d t \\
= & \frac{1}{n} \int_{1 / n}^{\pi} \psi_{x}\left(\frac{n t}{\lambda}\right) \frac{\cos n t}{t^{2}} d t+O\left(\omega_{2}\left(\frac{1}{n}, t\right)\right) \\
= & \frac{1}{n}\left\{\frac{\psi_{x}\left(\frac{n t}{\lambda}\right) \sin n t}{n t^{2}}\right\}_{1 / n}^{\pi}-\frac{1}{n^{2}} \int_{1 / n}^{\pi} \frac{\frac{n}{\lambda} \psi_{x}^{\prime}\left(\frac{n t}{\lambda}\right) t-2 \psi_{x}\left(\frac{n t}{\lambda}\right)}{t^{3}} \sin n t d t \\
& +O\left(\omega_{2}\left(\frac{1}{n}, t\right)\right) .
\end{aligned}
$$

It follows from (2.1) that 


$$
\begin{aligned}
\psi_{x}\left(\frac{n t}{\lambda}\right) & =P_{n}\left(x+\frac{n t}{\lambda}\right)+P_{n}\left(x-\frac{n t}{\lambda}\right)-2 P_{n}(x) \\
& =\frac{n^{2} t^{2}}{\lambda^{2}} P_{n}^{\prime \prime}(x+\theta t)=O\left(n^{2} t^{2} \omega_{2}\left(\frac{1}{n}, t\right)\right),
\end{aligned}
$$

$\psi_{x}^{\prime}\left(\frac{n t}{\lambda}\right)=P_{n}^{\prime}\left(x+\frac{n t}{\lambda}\right)-P_{n}^{\prime}\left(x-\frac{n t}{\lambda}\right)=\frac{2 n t}{\lambda} P_{n}^{\prime \prime}\left(x+\theta^{\prime} t\right)=O\left(n^{2} t \omega_{2}\left(\frac{1}{n}, f\right)\right)$,

$\psi_{n}^{\prime \prime}\left(\frac{n t}{\lambda}\right)=P_{n}^{\prime \prime}\left(x+\frac{n t}{\lambda}\right)+P_{n}^{\prime \prime}\left(x-\frac{n t}{\lambda}\right)=O\left(n^{2} \omega_{2}\left(\frac{1}{n}, f\right)\right)$.

Hence

$$
\begin{aligned}
A_{n}= & -\frac{1}{n^{2}} \int_{1 / n}^{\pi} \frac{\frac{n}{\lambda} \psi_{n}^{\prime}\left(\frac{n t}{\lambda}\right) t-2 \psi_{x}\left(\frac{n t}{\lambda}\right)}{t^{3}} \sin n t d t+O\left(\omega_{2}\left(\frac{1}{\lambda}, t\right)\right) \\
= & \left\{\frac{\frac{n}{\lambda} \psi_{x}^{\prime}\left(\frac{n t}{\lambda}\right) t-2 \psi_{x}\left(\frac{n t}{\lambda}\right)}{n^{3} t^{3}} \cos n t\right\}_{1 / n}^{\pi} \\
& -\frac{1}{n^{3}} \int_{1 / n}^{\pi}\left\{\frac{n^{2} t^{2}}{\lambda^{2}} \psi_{x}^{\prime \prime}\left(\frac{n t}{\lambda}\right)-\frac{n t}{\lambda} \psi_{x}^{\prime}\left(\frac{n t}{\lambda}\right)-6 \psi_{x}\left(\frac{n t}{\lambda}\right)\right. \\
& +O\left(\omega_{2}\left(\frac{1}{\lambda}, t\right)\right) \\
= & O\left(\omega_{2}\left(\frac{1}{\lambda}, t\right)\right) .
\end{aligned}
$$

By (2.2)

$$
\begin{aligned}
B_{n}= & \frac{1}{n} \int_{0}^{\pi} \cos n t \sum_{\nu=1}^{\infty} \frac{(-1)^{\nu n} \psi_{x}\left(\frac{\nu n \pi+n t}{\lambda}\right)}{(\nu \pi+t)^{2}} d t+O\left(\omega_{2}\left(\frac{1}{n}, f\right)\right) \\
= & \left.\frac{1}{n}\left\{\frac{\sin n t}{n} \sum_{\nu=1}^{\infty} \frac{\left.(-1)^{\nu n} \psi_{x}\left(\frac{\nu n \pi+n t}{\lambda}\right)\right\}_{0}^{\pi}}{(\nu \pi+t)^{2}}\right]_{0}^{n} \frac{\nu n \pi+n t}{\lambda}\right)(\nu \pi+t)-2(-1)^{\nu n} \psi_{x}\left(\frac{\nu n \pi+n t}{\lambda}\right) \\
& -\frac{1}{n^{2}} \int_{0}^{\pi} \sin n t \sum_{\nu=1}^{\infty} \frac{(-1)^{\nu n} \frac{(\nu+1)^{3}}{\lambda}}{(\nu \pi+t)^{3}} \\
& +O\left(\omega_{2}\left(\frac{1}{n}, f\right)\right) .
\end{aligned}
$$


Since $\psi_{x}^{\prime}$ and $\psi_{x}$ are periodic,

$$
\begin{aligned}
& \psi_{x}^{\prime}\left(\frac{v n \pi+n t}{\lambda}\right)=O\left(n^{2} \omega_{2}\left(\frac{1}{n}, f\right)\right), \\
& \psi_{x}\left(\frac{v n \pi+n t}{\lambda}\right)=O\left(n^{2} \omega_{2}\left(\frac{1}{n}, f\right)\right) .
\end{aligned}
$$

Hence

$$
B_{n}=O\left(\omega_{2}\left(\frac{1}{n}, f\right)\right)=O\left(\omega_{2}\left(\frac{1}{\lambda}, t\right)\right) .
$$

Thus lemma 1 is proved.

Lemma 2 . For $r \geqq 2$,

$$
\int_{1}^{\infty} \phi_{x}\left(\frac{t}{\lambda}\right) \frac{1-\cos t}{t^{2 r}} d t=O\left(\omega_{2}\left(\frac{1}{\lambda}, t\right)\right)
$$

Proof. Since, for $1 / n \leqq t \leqq \pi$,

$$
\begin{aligned}
\omega_{2}(t, f)= & O\left(n^{2} t^{2} \omega_{2}\left(\frac{1}{n}, f\right)\right), \\
\int_{1}^{\infty} \phi_{x}\left(\frac{t}{\lambda}\right) \frac{1-\cos t}{t^{2 r}} d t= & \frac{1}{n^{2 r-1}} \int_{1 / n}^{\pi} \phi_{x}\left(\frac{n t}{\lambda}\right) \frac{1-\cos n t}{t^{2 r}} d t \\
& +\frac{1}{n^{2 r-1}} \int_{0}^{\pi} \sum_{\nu=1}^{\infty} \frac{\left[1-(-1)^{\nu n} \cos n t\right] \phi_{x}\left(\frac{\nu n \pi+n t}{\lambda}\right)}{(\nu \pi+t)^{2}} d t \\
= & O\left(\frac{1}{n^{2 r-1}} \int_{1 / n}^{\pi} \frac{\left|\phi_{x}\left(\frac{n t}{\lambda}\right)\right|}{t^{2 r}} d t\right)+O\left(\omega_{2}\left(\frac{1}{n}, f\right)\right) \\
= & O\left(\frac{1}{n^{2 r-1}} \int_{1 / n}^{n} \frac{\omega_{2}(t, f)}{t^{2 r}} d t\right)+O\left(\omega_{2}\left(\frac{1}{n}, f\right)\right) \\
= & O\left(\frac{\omega_{2}\left(\frac{1}{n}, f\right)}{n^{2 r-3}} \int_{1 / n}^{\pi} \frac{d t}{t^{2 r-2}}\right)+O\left(\omega_{2}\left(\frac{1}{n}, f\right)\right) \\
= & O\left(\omega_{2}\left(\frac{1}{n}, f\right)\right)=O\left(\omega_{2}\left(\frac{1}{\lambda}, f\right)\right) .
\end{aligned}
$$

Thus Lemma 2 is proved.

By the same argument we can prove 
Lemma 3. For $r \geqq 2$,

$$
\begin{aligned}
& \int_{1}^{\infty} \phi_{x}\left(\frac{t}{\lambda}\right) \frac{\sin t}{t^{2 r-1}} d t=O\left(\omega_{2}\left(\frac{1}{\lambda}, f\right)\right), \\
& \int_{1}^{\infty} \phi_{x}\left(\frac{t}{\lambda}\right) \frac{\cos t}{t^{2 r}} d t=O\left(\omega_{2}\left(\frac{1}{\lambda}, f\right)\right) .
\end{aligned}
$$

\section{Proof of theorem A}

We have [1]

$$
\frac{1}{\pi} \int_{-\infty}^{\infty} f(x+t) \frac{\sin \lambda t}{t} d t=\sum_{\nu \leqq \lambda}^{\prime} s_{\nu}(x),
$$

where $\lambda$ is positive but not necessarily an integer, the integral is defined as $\lim _{T \rightarrow \infty} \int_{-T}^{T}$, and the dash indicates that if $\lambda$ is an integer then the last term of the sum is taken with a factor $\frac{1}{2}$.

Integrating with respect to $\lambda$ we verify by induction that

where

$$
\sum_{\nu \leqq \lambda}\left(1-\frac{\nu}{\lambda}\right)^{k} s_{\nu}(x)=\frac{1}{\pi} \int_{-\infty}^{\infty} f\left(x+\frac{t}{\lambda}\right) g(t) d t,
$$

$g(t)=\sum_{r=1}^{\left[\frac{k+1}{2}\right]}(-1)^{r}\left(\begin{array}{c}k \\ 2 r-1\end{array}\right) \frac{d^{(2 r-1)}}{d t^{(2 r-1)}}\left(\frac{1-\cos t}{t}\right)+\sum_{r=0}^{\left[\frac{k}{2}\right]}(-1)^{r}\left(\begin{array}{c}k \\ 2 r\end{array}\right) \frac{d^{(2 r)}}{d t^{(2 r)}}\left(\frac{\sin t}{t}\right)$, and so

$$
R_{\lambda}^{k}(x)-f(x)=\frac{1}{\pi} \int_{0}^{\infty} \phi_{x}\left(\frac{t}{\lambda}\right) g(t) d t .
$$

By expanding $g(t)$ into a power series of $t$, we know that it is uniformly bounded when $n$ is fixed and $0 \leqq t \leqq 1 / n$. Hence

$$
R_{\lambda}^{k}(x)-f(x)=\frac{1}{\pi} \int_{1}^{\infty} \phi_{x}\left(\frac{t}{\lambda}\right) g(t) d t+O\left(\omega_{2}\left(\frac{1}{\lambda}, f\right)\right) .
$$

By Leibniz' theorem,

$$
\begin{gathered}
(-1)^{r} \frac{d^{(2 r-1)}}{d t^{(2 r-1)}}\left(\frac{1-\cos t}{t}\right)=- \\
-\cdots+(-1)^{r-1}(2 r-1) ! \frac{\sin t}{t}-(2 r-1) \frac{\cos t}{t^{2}}+\frac{(2 r-1)(2 r-2)}{2 !} \frac{\sin t}{t^{2 r}} \\
(-1)^{r} \frac{d^{(2 r)}}{d t^{(2 r)}}\left(\frac{\sin t}{t}\right)=\frac{\sin t}{t}+2 r \frac{\cos t}{t^{2}}-\frac{2 r(2 r-1)}{2 !} \frac{\sin t}{t^{3}} \\
+\cdots+(-1)^{r}(2 r) ! \frac{\sin t}{t^{2 r+1}}
\end{gathered}
$$


and hence

$$
g(t)=\sum_{r=1}^{\left.\frac{k+1}{2}\right]}(-1)^{r-1}\left(\begin{array}{c}
k \\
2 r-1
\end{array}\right) \frac{1-\cos t}{t^{2 r}}+\sum_{r=1}^{\left[\frac{k}{2}\right]} p_{2 r}(k) \frac{\cos t}{t^{2 r}}+\sum_{r=1}^{\left[\frac{k}{2}\right]} p_{2 r-1} \frac{\sin t}{t^{2 r+1}},
$$

where $p_{1}(k), p_{2}(k), \cdots, p_{\left[\frac{k}{2}\right]}(k)$ are integers depending only on $k$. Thus by the lemmas,

$$
R_{\lambda}^{k}(x)-f(x)=\frac{k}{\pi} \int_{1}^{\infty} \frac{\phi_{x}\left(\frac{t}{\lambda}\right)}{t^{2}} d t+O\left(\omega_{2}\left(\frac{1}{\lambda}, f\right)\right) .
$$

Clearly we have

$$
\max _{|t| \leqq \max (1, a)}\left|\phi_{x}\left(\frac{t}{\lambda}\right)\right| \leqq \sup _{|t| \leqq \max (1, a)} \max _{x}\left|\phi_{x}\left(\frac{t}{\lambda}\right)\right|=O\left(\omega_{2}\left(\frac{1}{\lambda}, f\right)\right),
$$

and so

$$
\begin{aligned}
\left|\int_{a}^{1} \frac{\phi_{x}(t / \lambda)}{t^{2}} d t\right| \leqq\left|\int_{a}^{1} \frac{\left|\phi_{x}(t / \lambda)\right|}{t^{2}} d t\right| & =O\left(\omega_{2}\left(\frac{1}{\lambda}, f\right) \int_{a}^{1} \frac{d t}{t^{2}}\right) \\
& =O\left(\omega_{2}\left(\frac{1}{\lambda}, f\right)\right) .
\end{aligned}
$$

Finally,

$$
R_{\lambda}^{k}(x)-f(x)=\frac{k}{\pi} \int_{a}^{\infty} \frac{\phi_{x}(t / \lambda)}{t^{2}} d t+O\left(\omega_{2}\left(\frac{1}{\lambda}, f\right)\right)
$$

and the theorem is therefore proved.

\section{References}

[1] A. Zygmund, Trigonometric series (Cambridge, 1959).

[2] S. B. Steckin, 'On the order of the best approximations of continuous functions', $I z v$. Akad. Nauk USSR Ser. Mat. 15 (1951), 219-242 (Russian).

Department of Mathematics

University of Malaya

Kuala Lumpur, Malaysia 